\title{
AUTOIMMUNITY
}

\section{Pinpointing progression to T1DM in children at genetic risk}

New data reveal that in children at genetic risk of type 1 diabetes mellitus (T1DM) who progress to clinical disease before puberty, the diabetes-associated autoimmune process starts before the age of 4 years.

"We searched for the earliest possible predictive patterns of future clinical disease in children with HLA-conferred genetic risk of T1DM," explains lead researcher Vilhelmiina Parikka of Turku University Hospital, Finland. "A 3-monthly sampling interval during the first years of life enabled us to pinpoint not only the time of seroconversion but also the sequence of the appearance of the individual autoantibodies."

The study included 1,320 children at genetic risk of T1DM who seroconverted to autoantibody positivity during a median follow-up of 7.7 years. Whether the children were positive for four major diabetes-associated autoantibodies was assessed at 3-12 month intervals from birth; in a subcohort, sampling occurred at 3-monthly intervals until 2 years of age.

During follow-up, 184 children progressed to T1DM. Of these, age at seroconversion was $<2$ years in $118(64 \%)$ and $<3$ years in 150 (82\%). Incidence of seroconversion peaked at 1 year of age and was more likely to happen at 12-17 months of age than at 18-23 months of age. Initial seroconversion progressed to multiple autoantibody positivity within 1 year in children who later developed T1DM. Autoantibody concentration was also important. A marked increase in autoantibody concentration during the first few months following initial seroconversion was identified as a strong predictor of progression to T1DM.

Parikka comments that the findings indicate the importance of very early life events in the disease process and suggest that triggers of diabetes-associated autoimmunity should be searched for in at-risk children $\leq 1$ year old. The team now plan to investigate the environmental factors associated with this early start of autoimmunity.

According to George Eisenbarth of the University of Colorado, who was not involved in the study, "even 3-month intervals of testing, as in this great study, may be too infrequent to find environmental factors that suddenly trigger appearance of islet autoantibodies." He suggests that "development of pointof-care, nonradioactive islet autoantibody assays might enable weekly or even daily follow-up, for studies of the first 2 years of life."

Is prevention of $\mathrm{T} 1 \mathrm{DM}$ on the horizon? "Ultimately," Eisenbarth concludes, "the ability to accurately predict T1DM provides the opportunity to prevent the disorder. Trials to date I believe have utilized relatively 'blunt' therapeutics, given the increasing understanding of molecular pathogenesis. The combination of improved basic knowledge of immune pathogenesis and the disease's natural history will most likely lead to safe prevention in the future."

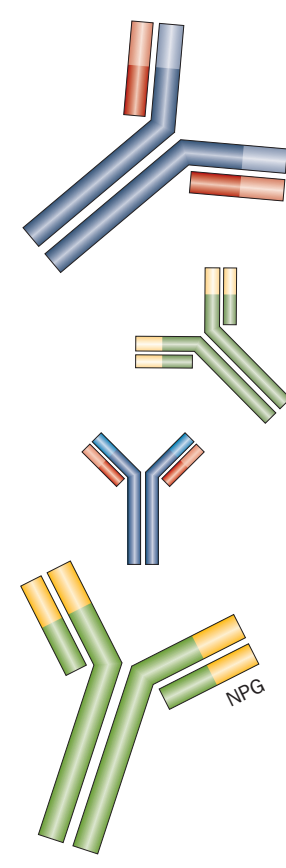

Carol Wilson

Original article Parikka, V. et al. Early seroconversion and rapidly increasing autoantibody concentrations predict prepubertal manifestation of type 1 diabetes in children at genetic risk. Diabetologia doi:10.1007/s00125-012 2523-3 